\title{
A mass spectrometry-based method for the determination of in vivo biodistribution of tumor targeting small molecule-metal conjugates
}

Ettore Gilardoni ${ }^{1}$, Aureliano Zana ${ }^{\S 1}$, Andrea Galbiati ${ }^{1}$, Theo Sturm ${ }^{1}$, Jacopo Millul ${ }^{1}$, Samuele Cazzamalli ${ }^{1}$, Dario Neri2,3* , Riccardo Stucchi ${ }^{1 *}$

${ }^{1}$ Philochem AG, Libernstrasse 3, CH-8112, Otelfingen, ZH, Switzerland

${ }^{2}$ Philogen S.P.A. Via Bellaria 35, I-53018 Sovicille, SI, Italy

${ }^{3}$ Swiss Federal Institute of Technology, Department of Chemistry and Applied Biosciences, $\mathrm{CH}-8093$ Zurich, Switzerland

$\S$ Authors contributed equally to this work

*Corresponding author information:

Riccardo Stucchi - riccardo.stucchi@philochem.ch

Dario Neri - dario.neri@pharma.ethz.ch 


\section{Abbreviations:}

ACN:

Acetonitrile

DOTAGA:

2-(4,7,10-tris(carboxymethyl)-1,4,7,10-tetraazacyclododecan-1yl)pentanedioic acid

EDTA: $\quad$ Ethylenediaminetetraacetic acid

FA:

Formic acid

FAP:

Fibroblast activation protein

HPLC:

High pressure liquid chromatography

HRMS:

High resolution mass spectrometry

ICP-MS:

Inductively coupled plasma mass spectrometry

ID/g:

Injected dose per gram

IS:

Internal standard

LC:

Liquid chromatography

LC-MS:

Liquid chromatography - Mass spectrometry

MS:

Mass spectrometry

NODAGA:

2-(4,7-bis(carboxymethyl)-1,4,7-triazonan-1-yl)pentanedioic acid

NOTA:

2,2'-(7-carboxy-1,4,7-triazonane-1,4-diyl)diacetic acid

PBS:

Phosphate-buffered saline

SIM:

Single ion monitoring

SMMC:

Small molecule metal conjugate

SMRC:

Small molecule radio conjugate

SPE:

Solid phase extraction

TFA:

Trifluoroacetic acid 


\section{Abstract}

Nuclear medicine plays a key role in modern diagnosis and cancer therapy. The development of tumor targeting radionuclide conjugates (also named Small Molecule-Radio Conjugates SMRCs) represents a significant improvement over the clinical use of metabolic radiotracers (e.g., $\left[{ }^{18} \mathrm{~F}\right]$-Fluorodeoxyglucose) for imaging and over the application of biocidal external beam radiations for therapy. During the discovery of SMRCs, molecular candidates must be carefully evaluated typically by performing biodistribution assays in preclinical tumor models. Quantification methodologies based on radioactive counts are typically demanding due to safety concerns, availability of radioactive material, and infrastructures. In this article, we report the development of a mass spectrometry (MS)-based method for the detection and quantification of small molecule-metal conjugates (SMMCs) as cold surrogates of SMRCs. We applied this methodology for the evaluation of the biodistribution of a particular class of tumortargeting drug candidates based on ${ }^{n a t} \mathrm{Lu},{ }^{n a t} \mathrm{Ga}$, nat $F$ and directed against Fibroblast Activation Protein (FAP). The reliability of the LC-MS analysis was validated by direct comparison of MSbased and radioactivity-based biodistribution data. Results show that MS biodistribution of stable isotope metal conjugates is an orthogonal tool for the preclinical characterization of different classes of radiopharmaceuticals.

\section{Introduction}

Small molecule-radio conjugates (SMRCs) are radioactive compounds which typically consist of two components: a radionuclide included in a molecular structure and a targeting moiety which is responsible for specific accumulation of the radioactive payload the site of disease (typically cancer lesions) ${ }^{1}$. Thanks to this peculiar design, SMRCs find applications both as diagnostic tracers and as radiotherapeutic agents in modern nuclear medicine. In the recent years, clinical success of Lutathera ${ }^{2}{ }^{2}$ and of PSMA- $617^{3}$ for the treatment of patients with neuroendocrine tumors and metastatic castration resistant prostate cancer has demonstrated the potential of this class of theragnostic. Many novel SMRC drug candidates are being developed and studied in clinical trials for diagnostic and therapeutic applications of patients with cancer and other types of chronic malignancies ${ }^{4,5}$,

Among several new tumor-targeting radiopharmaceuticals, SMRCs targeting fibroblast activation protein (FAP) are promising pan-tumoral compounds which accumulate to tumors with exquisite selectivity against normal tissues ${ }^{4,6,7}$. FAP is a membrane-bound serine protease abundantly expressed on the stroma of most epithelial cancers ${ }^{8}$. FAP has been validated as high-quality tumor-associated antigen by immunohistochemistry and nuclear medicine ${ }^{4,6,7,9}$. Our group has recently developed OncoFAP, a small molecule targeting FAP 
with ultra-high affinity $\left(\mathrm{K}_{\mathrm{D}}=680 \mathrm{pM}\right)$, able to selectively deliver radionuclides, such as Lutetium and Gallium, to the tumor site ${ }^{10}$. A Gallium-68 OncoFAP derivative shows excellent tumortargeting properties in patients with solid malignancies, with tumor-to-organ ratio of $\sim 20$-to-1 at early time points ${ }^{6}$. This compound represents an ideal candidate for imaging applications. More recently, we developed a dimeric derivative owing two targeting moieties (i.e., BiOncoFAP), showing higher tumor uptake and residence time than OncoFAP in preclinical models of cancer ${ }^{11}$.

The biodistribution properties of novel tumor targeting SMRCs are of key importance for the success of the diagnosis and therapeutic outcome. Preclinical biodistribution of radiopharmaceuticals in tumor-bearing animals is typically evaluated by direct measurement of the radioactivity present in each organ after sacrifice of experimental animals ${ }^{10,11}$. To date, these radioactivity-based analyses are still crucial to evaluate the tumor accumulation and to obtain spatial biodistribution data of the compounds in the animal/human body. On the contrary, for most of the other non-radioactive pharmaceuticals, LC-MS analysis represents the analytical method of choice when it comes to accurately quantify the analyte of interest in biological specimens ${ }^{12,13}$. Since radioactivity-based experiments are challenging because of safety reasons (i.e., potential exposure of the operator to harmful radiation doses) and availability of dedicated infrastructures, the development of an alternative analytical methodology would open exciting new possibilities.

To our knowledge, most of the studies reported in the literature for the analysis of nonradioactive metal-conjugates rely on HPLC-UV or ICP-MS methods ${ }^{14}$, while only a very limited number exploit LC-MS techniques ${ }^{15,16}$.

In this work, we developed a MS-based quantification methodology which allows to determine quantitative biodistribution of Small molecule-metal conjugates (SMMCs) surrogates of SMRCs based on cold non-radioactive isotopes, aiming at offering a valid alternative to the use of hot radionuclides throughout the discovery and development of radiopharmaceuticals. By comparing "cold" biodistribution data (i.e., data obtained after administration of SMMCs) obtained by mass spectrometry and radio-biodistribution data (i.e., data obtained after administration of the corresponding SMRCs), we validated this novel LC-MS method as an orthogonal, safe, green, and easy-to-implement option to classical radioactivity-based methodologies. 


\section{Experimental Section}

\section{Chemicals and Materials}

All reagents and solvents were purchased from Sigma Aldrich, VWR, Combi-Blocks, CheMatech and used as supplied.

\section{Chemical Synthesis}

OncoFAP and BiOncoFAP, as well as their metal chelator conjugates and "cold"-labeled derivatives were synthesized through established protocols $\mathrm{s}^{6,10,11}$.

For the synthesis of internal standards (ISs), we used isotopically labeled building blocks. In particular, ${ }^{13} \mathrm{C}_{4}$-succinic anhydride was exploited for OncoFAP derivatives, while ${ }^{13} \mathrm{C}_{6}{ }^{15} \mathrm{~N}_{2}$-LLysine was used for BiOncoFAP-conjugates.

Detailed experimental chemical procedures are described in the Supplemental Informations.

\section{Animal studies}

All animal experiments were conducted in accordance with Swiss animal welfare laws and regulations under the license number ZH006/2021 granted by the Veterinäramt des Kantons Zürich.

In vivo biodistribution of OncoFAP molecules in tumor-bearing mice

Tumor cells (HT-1080.hFAP, or SK-RC-52.hFAP cells) were grown to $80 \%$ confluence and detached with Trypsin-EDTA 0.05\% (Gibco). Cells were then resuspended in Hanks' Balanced Salt Solution medium (Gibco). Aliquots of 5 to $10 \times 10^{6}$ cells (100 to $150 \mu \mathrm{L}$ of suspension) were injected subcutaneously in the right flanks of female athymic Balb/c AnNRj-Foxn1 mice (6 to 8 weeks of age, Janvier).

Mice bearing subcutaneous HT-1080.hFAP tumors were injected intravenously with [ ${ }^{\text {nat }} \mathrm{Lu}$ ]Lu-

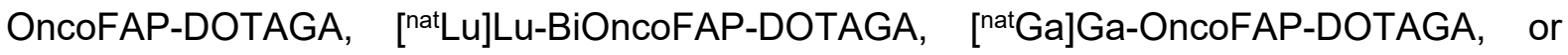
[ ${ }^{\text {nat }} \mathrm{Ga}$ ]Ga-BiOncoFAP-DOTAGA (5 nmol dissolved in sterile PBS, $\mathrm{pH} 7.4$ ). Animals were sacrificed $1 \mathrm{~h}$ after intravenous injection. Fresh blood was collected in lithium heparin tubes (BD Microcontainer LH Tubes), vortexed and centrifuged (15’000 g, $15 \mathrm{~min}$ ). Plasma was frozen and stored at $-80^{\circ} \mathrm{C}$. Healthy organs and tumors were subsequently excised, frozen with dry ice and stored at $-80^{\circ} \mathrm{C}$. 
Mice bearing subcutaneous SK-RC-52.hFAP tumors were injected intravenously with

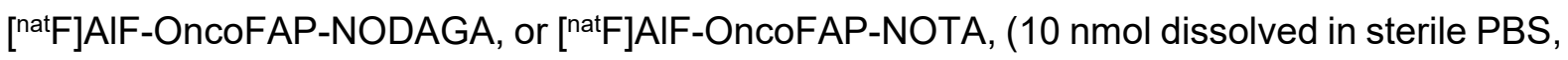
$\mathrm{pH}$ 7.4). Animals were sacrificed $2 \mathrm{~h}$ after intravenous injection. Fresh blood was collected in lithium heparin tubes (BD Microcontainer LH Tubes), vortexed and centrifuged (15'000 g, 15 $\min )$. Plasma was frozen and stored at $-80^{\circ} \mathrm{C}$. Healthy organs and tumors were subsequently excised, frozen with dry ice and stored at $-80^{\circ} \mathrm{C}$

Sample preparation for Mass Spectrometry analysis

$50 \mathrm{mg}$ of mice tissues were resuspended in $600 \mu \mathrm{L}$ of a solution containing $95 \% \mathrm{ACN}$ and $0.1 \% \mathrm{FA}$ to induce protein precipitation. In parallel, $50 \mu \mathrm{L}$ of a solution $600 \mathrm{nM}$ of internal standard ([ ${ }^{\text {nat } L u] L u-~}{ }^{13} \mathrm{C}_{4}$-OncoFAP-DOTAGA, or [ ${ }^{\text {natLu}} \mathrm{Lu}-{ }^{13} \mathrm{C}_{6}{ }^{15} \mathrm{~N}_{2}$-BiOncoFAP-DOTAGA, or [ ${ }^{\text {nat }} \mathrm{Ga}$ ]Ga- ${ }^{13} \mathrm{C}_{4}$-OncoFAP-DOTAGA, or [ ${ }^{\text {nat }} \mathrm{Ga}$ ] Ga- ${ }^{13} \mathrm{C}_{6}{ }^{15} \mathrm{~N}_{2}$-BiOncoFAP-DOTAGA, or [ ${ }^{\text {nat }} \mathrm{F}$ ]AIF${ }^{13} \mathrm{C}_{4}$-OncoFAP-NODAGA, or $\left[{ }^{\text {nat }} \mathrm{F}\right] A \mathrm{AIF}-{ }^{13} \mathrm{C}_{4}$-OncoFAP-NOTA) were added to the mixture. Samples were then homogenized with a tissue lyser (TissueLyser II, QIAGEN) for 15 minutes at $30 \mathrm{~Hz}$. After homogenization, samples were centrifugated at $15^{\prime} 000 \mathrm{~g}$ for 10 minutes and supernatants were dried at room temperature with a vacuum centrifuge (Eppendorf). Pellets were then resuspended in $1 \mathrm{~mL}$ solution containing $3 \% \mathrm{ACN}$ and $0.1 \%$ of TFA and subsequently purified on Oasis HLB SPE columns (Waters) following instructions indicated by the manufacturer. Eluates were dried under vacuum at room temperature, resuspended in 400 $\mu \mathrm{L} 3 \% \mathrm{ACN}$ and $0.1 \%$ of TFA and further purified on Macrospin column (Harvard Apparatus). Eluates were then dried under vacuum at room temperature.

Dried samples were finally resuspended in $30 \mu \mathrm{L}$ of a solution containing $3 \%$ of $\mathrm{ACN}$ and $0.1 \%$ of FA. Each sample $(1.5 \mu \mathrm{L}, 5 \%$ of the total) was then injected in the nanoLC-HRMS system. nanoLC-HRMS analysis

Chromatographic separation was carried out on an Acclaim PepMap RSLC column (50 $\mu \mathrm{m} x$ $15 \mathrm{~cm}$, particle size $2 \mu \mathrm{m}$, pore size $100 \AA$, Thermo Fisher Scientific) with a gradient program from $95 \% \mathrm{~A}\left(\mathrm{H}_{2} \mathrm{O}, 0.1 \% \mathrm{FA}\right), 5 \% \mathrm{~B}(\mathrm{ACN} 0.1 \% \mathrm{FA})$ to $5 \% \mathrm{~A}, 95 \% \mathrm{~B}$ in 45 minutes on an Easy nanoLC 1000 (Thermo Fisher Scientific). The LC system was coupled to a Q-Exactive mass spectrometer (Thermo Fisher Scientific) via a Nano Flex ion source (Thermo Fisher Scientific). Ionization was carried out with $2 \mathrm{kV}$ of spray voltage, $250{ }^{\circ} \mathrm{C}$ of capillary temperature, $60 \mathrm{~S}$ lens RF level. The mass spectrometer was operating in Single lon Monitoring (SIM) mode, following the mass range reported in Table 1. The detector was working in positive ion mode with the following parameters: resolution 70000 (FWHM at $200 \mathrm{~m} / \mathrm{z}$ ), AGC target $5 \times 10^{4}$, and maximum injection time $200 \mathrm{~ms}$. 


\begin{tabular}{|c|c|}
\hline Compound & Mass Range (m/z) \\
\hline [ ${ }^{\text {nat }}$ Lu]Lu-OncoFAP-DOTAGA, $\left[{ }^{\text {nat }}\right.$ Lu]Lu$-{ }^{13} \mathbf{C}_{4}-$ OncoFAP -DOTAGA & $565.6634-573.6634$ \\
\hline [natLu]Lu-BiOncoFAP-DOTAGA, $\left[{ }^{\text {nat }} \mathrm{Lu}\right] \mathrm{Lu}-{ }^{13} \mathrm{C}_{6}{ }^{15} \mathrm{~N}_{2}$-BiOncoFAP-DOTAGA & $850.27326-58.27326$ \\
\hline 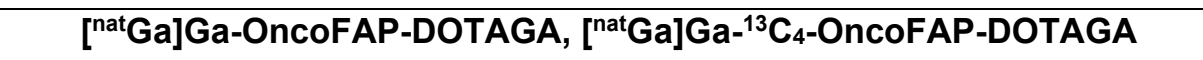 & $512.6557-520.6557$ \\
\hline [ ${ }^{\text {nat } G a] G a-B i O n c o F A P-D O T A G A, ~}\left[{ }^{\text {nat }}\right.$ Ga]Ga- ${ }^{13} \mathrm{C}_{6}{ }^{15} \mathrm{~N}_{2}$-BiOncoFAP-DOTAGA & $797.2657-805.2657$ \\
\hline$\left[{ }^{\text {nat }}\right.$ F]AIF-OncoFAP-NODAGA, $\left[{ }^{\text {nat }} \mathrm{F}\right] A I F-{ }^{13} \mathrm{C}_{4}$ OncoFAP-NODAGA & $901.8188-910.8188$ \\
\hline Al-OncoFAP-NODAGA, $\mathrm{Al}^{-13} \mathrm{C}_{4}-$-OncoFAP-NODAGA & $881.8126-890.8126$ \\
\hline AIOH-OncoFAP-NODAGA, AIOH- ${ }^{13} \mathrm{C}_{4}-\mathrm{O}$-OncoFAP-NODAGA & $899.8208-908.8208$ \\
\hline 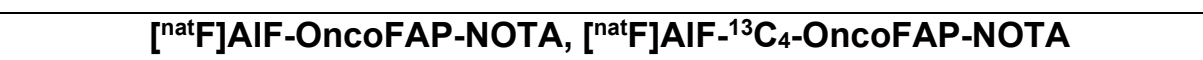 & $855.8133-864.8133$ \\
\hline
\end{tabular}

Table 1: mass range windows for the SIM mode of the mass spectrometer.

Data analysis

Peak areas of analytes and internal standards were integrated, and corresponding ratios calculated. Ratios were then transformed in $\mathrm{pmol} / \mathrm{g}$ of wet tissue using single concentration external calibration points (Table S1) and corrected by the total weight of the sample analyzed. The percentage of injected dose per gram $(\% / D / g)$ was finally determined by normalizing the value based on the total dose injected in the mouse (i.e., $5 \mathrm{nmol}$, or $10 \mathrm{nmol}$ ). All biodistribution experiments were performed using 3 mice per experimental condition. Graphs express mean \pm standard deviation values. Multiple t-test was done to compare $\% I D / g$ in each organ for i) [ ${ }^{\text {nat } L u] L u-O n c o F A P-D O T A G A}$ and [ [77Lu]Lu-OncoFAP-DOTAGA; ii) [ ${ }^{\text {nat } L u] L u-B i O n c o F A P-}$ DOTAGA and [177Lu]Lu-BiOncoFAP-DOTAGA; iii) [natGa]Ga-OncoFAP-DOTAGA and

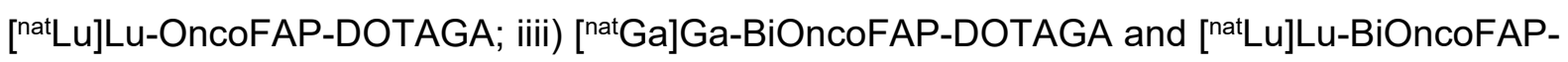
DOTAGA. Data analysis was performed with Thermo Xcalibur Qual Broswer v2.2 (Thermo Fisher Scientific) and Prism8 (GraphPad). 


\section{Results and Discussion}

\section{Method Development}

We have developed an innovative LC-MS method to measure SMMCs targeting FAP in invivo biodistribution experiments. Figure 1 presents a summary of the methodology, the schematic workflow of the experimental design, and the sample preparation procedure. The method was applied for the quantification of OncoFAP-derivatives (Figure 2) in biological

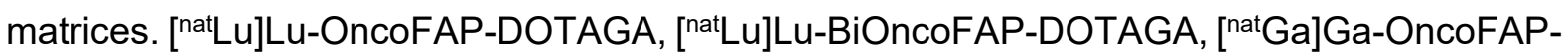

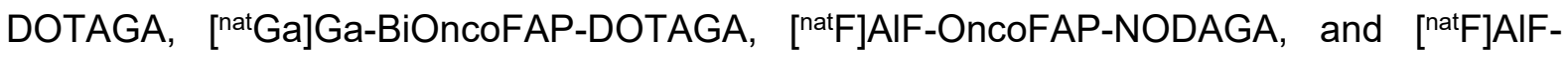
OncoFAP-NOTA were obtained in high purity and yields following well-established and previously described procedures ${ }^{11}$ (Supplementary Info).

Ionization efficiency and chromatography parameters (e.g., peak shape, resolution, and retention time) were evaluated for each compound by injecting an aqueous solution in the LCMS system and method parameters were tuned accordingly. Sample preparation was developed with the purpose to maximize the enrichment of the analytes after extraction from biological matrices prior to the quantification by LC-MS (Figure 1). Matrix effect and recovery after sample preparation were evaluated by spiking known amounts of the analytes in SK-RC52 or HT-1080 tumor tissues from untreated animals ("blank" tumors) (Table S2). Matrix effect was found to be variable between different analytes, with a $90 \%$ loss of signal for DOTAGAconjugates and $\sim 50 \%$ for NOTA and NODAGA derivatives. A recovery of $\sim 50 \%$ was instead observed for all analyzed compounds.

Due to significant loss of signal observed and to obtain a reliable quantification, we decided to include highly pure and stable isotopically labeled internal standards (ISs) before sample preparation. ISs were synthesized following identical synthetic routes utilized for the analytes, replacing key reagents with corresponding ${ }^{13} \mathrm{C}$ or ${ }^{15} \mathrm{~N}$ labeled compounds. ${ }^{13} \mathrm{C}_{4}$ succinic anhydride was used as building block for OncoFAP-based molecules, while ${ }^{13} \mathrm{C}_{6}{ }^{15} \mathrm{~N}_{2}$-L-Lysine was used for BiOncoFAP derivatives (Figure 2). Sensitivity of the analysis was further improved by operating the MS instrument in SIM mode. The simultaneous detection of analytes and of corresponding ISs in the same scan range allowed to minimize the interference caused by co-elution of contaminants. The increased sensitivity of the SIM mode and the use of isotopically labelled ISs allowed us to detect and precisely measure the analytes in all tissues analyzed despite the not complete recovery after sample preparation, and the high matrix effects for some derivatives. 


\section{Biodistribution of [nat Lu]Lu-OncoFAP-DOTAGA and [ ${ }^{\text {mat } L u] L u-B i O n c o F A P-D O T A G A}$}

We have recently presented biodistribution results obtained with $\left[{ }^{177} \mathrm{Lu}\right] \mathrm{Lu}-$ OncoFAPDOTAGA and [177Lu]Lu-BiOncoFAP-DOTAGA in HT-1080.hFAP tumor bearing mice that show favorable and selective accumulation of both compounds in FAP-positive tumors ${ }^{11}$. With the aim of benchmarking our LC-MS methodology with radioactivity-based results obtained with $\left[{ }^{177} \mathrm{Lu}\right]$ Lu-OncoFAP-DOTAGA and $\left[{ }^{177} \mathrm{Lu}\right]$ Lu-BiOncoFAP-DOTAGA, we studied quantitative biodistributions of their cold counterparts. Mice bearing HT-1080.hFAP tumors were treated with [ ${ }^{\text {nat }} \mathrm{Lu}$ ]Lu-OncoFAP-DOTAGA and [ ${ }^{\text {nat } L u] L u-B i O n c o F A P-D O T A G A}$ and sacrificed 1 hour after injection. Sensitivity of LC-MS analysis was high enough to accurately measure both compounds in any specimen analyzed. As expected, both molecules accumulated selectively in FAP-positive tumors. The BiOncoFAP product exhibited a higher tumor uptake as compared to its monovalent counterpart (Figures 3, Table S3). Uptake values in tumor and healthy organs (in \%ID/g) measured by the LC-MS methodology were comparable to values obtained with radioactivity-based quantification ${ }^{11}$. No significant differences were observed between the two analytical techniques ( $p>0.05$ multiple $t$ test, Table S4) (Figures 3). Orthogonal validation further confirms the accuracy of the newly developed MS quantification method, thereby opening new opportunities for non-radioactive LC-MS analysis of SMMCs.

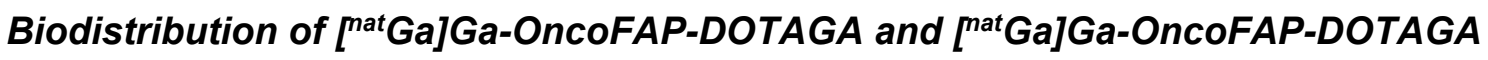

Encouraged by these results, we further applied our methodology for the assessment of the biodistributions of [nat $G a] G a-O n c o F A P-D O T A G A$ and [ ${ }^{\text {nat }} \mathrm{Ga}$ ]Ga-BiOncoFAP-DOTAGA, cold molecular counterparts of two novel diagnostic radiotracers (i.e., [ ${ }^{68} \mathrm{Ga}$ Ga-OncoFAPDOTAGA and $\left.\left[{ }^{68} \mathrm{Ga}\right] \mathrm{Ga}-B i O n c o F A P-D O T A G A\right) 6$ (Figure 4, table S5). Similarly to Lutetium derivatives, both compounds showed a consistent tumor uptake and no significant uptake in healthy organs, with favorable tumor-to-organ ratios. Uptake values obtained for [ $\left.{ }^{\text {nat }} \mathrm{Ga}\right] \mathrm{Ga}-$ OncoFAP-DOTAGA and [ ${ }^{\text {nat }} \mathrm{Ga}$ ]Ga-BiOncoFAP-DOTAGA showed no significant differences with [ ${ }^{\text {nat }} \mathrm{Lu}$ ] derivatives ( $p>0.05$ multiple $t$ test, table S4), except for a lower liver uptake observed for both ${ }^{\text {nat } G a}$ counterparts ( $p<0.05$ multiple $t$ test table $\mathbf{S 4}$ ). Despite some minor differences, probably caused by the intrinsic physical-chemical properties of the molecules, the tumor accumulations of the mono and bivalent OncoFAP-metal-conjugates were remarkable in every biodistribution experiment performed. Our results further confirm the versatility of this class of molecules as targeted radiopharmaceuticals $6,10,11$. 


\section{Biodistribution of [natF]AIF-OncoFAP-NODAGA and [ ${ }^{\text {nat }}$ F]AIF-OncoFAP-NOTA}

Besides Gallium-68 and Lutetium-177, Fluorine-18 is also frequently used for nuclear medicine applications in cancer patients ${ }^{17-19}$. Fluorine-18 can be covalently bound to tumor targeting agents (i.e., $\left[{ }^{18} \mathrm{~F}\right.$-Fluorodeoxyglucose) ${ }^{20}$ or indirectly incorporated as counter-ion in Aluminum complexes ${ }^{19}$. Among several metal chelators commercially available for chelating Aluminum Fluoride, the most widely used are NODAGA and NOTA ${ }^{19,21,22}$. For this purpose, OncoFAP-NODAGA and OncoFAP-NOTA were synthetized and labeled with [ ${ }^{\text {natF]AIF }}$. After

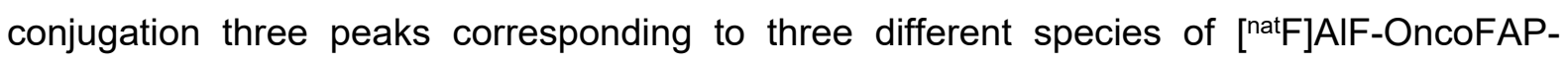
NODAGA were identified by MS (Figure 5A), while only one peak was observed for [ ${ }^{\text {natF]AIF- }}$ OncoFAP-NOTA (Figure 5B). The three peaks of [natF]AIF-OncoFAP-NODAGA could be unambiguously assigned to AIOH-OncoFAP-NODAGA ( $\mathrm{Rt}=13.56 \mathrm{~min}$ ), Al-OncoFAPNODAGA ( $R t=15.34 \mathrm{~min})$ and [ ${ }^{\text {natF}}$ ]AIF-OncoFAP-NODAGA $(\mathrm{Rt}=16.42 \mathrm{~min})$ (Figure 5A). Among the three species present, the most abundant one corresponds to Al-OncoFAPNODAGA. This observation is not unexpected since it is known that NODAGA $\mathrm{N}_{3} \mathrm{O}_{3}$ configuration is not favorable for $\mathrm{AlF}_{3}$ chelation ${ }^{18,19}$. By observing the structures of NODAGA and NOTA chelators, it is possible to note the presence of an extra carboxylic group in the NODAGA structures that saturates Aluminum coordination sphere ${ }^{18,22}$, resulting in the loss of the Fluoride anion (Figure S1). On the contrary, NOTA $\mathrm{N}_{3} \mathrm{O}_{2}$ configuration, facilitates the $\mathrm{AlF}_{3}$ chelation not having a carboxylic group competing with the Fluoride interaction ${ }^{18,23}$ (Figure S1). Biodistribution experiments were conducted in SK-RC-52.hFAP bearing mice treated with

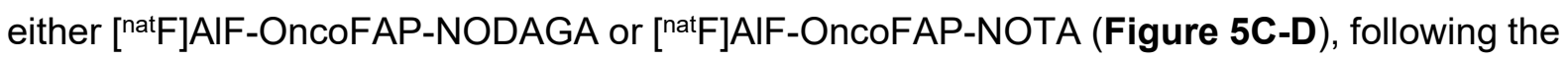
same technical procedures and quantification methods described above. The $\% \mathrm{ID} / \mathrm{g}$ of OncoFAP-NODAGA was calculated as sum of the three different molecular species since it was not possible to separate them after cold-labeling and therefore a mixture of the three was injected in the animals. Our newly developed LC-MS methodology allowed us to precisely follow the biodistribution of each single molecular species of OncoFAP-NODAGA, thereby resulting into a more accurate analysis than classical radioactive-based method which are limited to the $\left[{ }^{18} \mathrm{~F}\right]$ AIF-OncoFAP-NODAGA only species (Figure S2, Table S6). Notably, both molecules are characterized by favorable tumor-to-organ ratios and do not show any

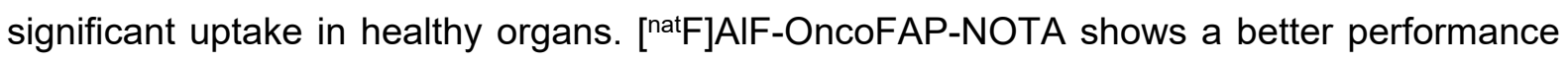
since it exhibited a higher tumor uptake compared to OncoFAP-NODAGA (i.e., $12.83 \pm 0.87$ vs $5.19 \pm 1.55 \%$ ID/g.) (Figure 5C-D. Table S7). 


\section{Conclusion}

In this article, we have reported the development and the application of a novel quantitative LC-MS methodology for the assessment of the in vivo biodistribution analysis of tumor targeting SMMCs. The method presented here is reliable and accurate in providing biodistribution data which are fully comparable to those obtained by radioactive-based experiments. The use of stable isotopes and the successful quantification of SMMCs by MS represent a valid alternative to classical approaches, opening new possibilities in the discovery of novel radiopharmaceuticals. Quantification methodologies based on radioactivity are often used and can be very accurate, if a suitable radiolabeling procedure is used. However, regulatory constraints (e.g., the need for dedicated laboratories and infrastructure) as well as safety concerns (e.g., exposure of the operator to harmful radiations) limit the applicability of radioactivity-based methodologies, both in academia and in industry. In this context, our newly developed LC-MS methodology represents a valuable orthogonal, safe, green, and easy-toimplement alternative. Our vision considers further integration of LC-MS quantification in drug development of targeted SMRCs. We envisage a further expansion of the technology described in this article for the assessment of in vivo biodistribution of other classes of targeted drugs (e.g., Small Molecule-Drug Conjugates and antibody-cytokine fusion proteins). Overall, the reduction of the radioactive or cytotoxic compounds during discovery phases will translate into an enhanced throughput and, ultimately, into higher possibilities of identification of novel clinical candidates.

\section{Acknowledgment}

Disclosure: D.N. is a cofounder and shareholder of Philogen S.p.A. (http://www.philogen.com/en/), a Swiss-Italian Biotech company that operates in the field of ligand-based pharmacodelivery. E.G., A.Z, A.G., T.S., J.M, S.C, and R.S. are employees of Philochem AG, the daughter company of Philogen, that owns and has patented OncoFAP, BiOncoFAP and their derivatives. No other potential conflicts of interest relevant to this article exist. 


\section{References}

(1) Dal Corso, A. Targeted Small-Molecule Conjugates: The Future Is Now. ChemBioChem 2020, 21 (23), 3321-3322. https://doi.org/https://doi.org/10.1002/cbic.202000507.

(2) Hennrich, U.; Kopka, K. Lutathera®: The First FDA- and EMA-Approved Radiopharmaceutical for Peptide Receptor Radionuclide Therapy. Pharmaceuticals 2019, 12 (3). https://doi.org/10.3390/ph12030114.

(3) Sartor, O.; de Bono, J.; Chi, K. N.; Fizazi, K.; Herrmann, K.; Rahbar, K.; Tagawa, S. T.; Nordquist, L. T.; Vaishampayan, N.; El-Haddad, G.; Park, C. H.; Beer, T. M.; Armour, A.; Pérez-Contreras, W. J.; DeSilvio, M.; Kpamegan, E.; Gericke, G.; Messmann, R. A.; Morris, M. J.; Krause, B. J. Lutetium-177-PSMA-617 for Metastatic CastrationResistant Prostate Cancer. New England Journal of Medicine 2021, 385 (12), 10911103. https://doi.org/10.1056/NEJMoa2107322.

(4) Herrmann, K.; Schwaiger, M.; Lewis, J. S.; Solomon, S. B.; McNeil, B. J.; Baumann, M.; Gambhir, S. S.; Hricak, H.; Weissleder, R. Radiotheranostics: A Roadmap for Future Development. The Lancet Oncology 2020, $21 \quad$ (3), e146-e156. https://doi.org/10.1016/S1470-2045(19)30821-6.

(5) Kunos, C. A.; Mankoff, D. A.; Schultz, M. K.; Graves, S. A.; Pryma, D. A. Radiopharmaceutical Chemistry and Drug Development-What's Changed? Seminars in Radiation Oncology 2021, $31 \quad$ (1), 3-11. https://doi.org/https://doi.org/10.1016/j.semradonc.2020.07.006.

(6) Backhaus, P.; Gierse, F.; Burg, M. C.; Büther, F.; Asmus, I.; Dorten, P.; Cufe, J.; Roll, W.; Neri, D.; Cazzamalli, S.; Millul, J.; Mock, J.; Galbiati, A.; Zana, A.; Schäfers, K. P.; Hermann, S.; Weckesser, M.; Tio, J.; Wagner, S.; Breyholz, H.-J.; Schäfers, M. Translational Imaging of the Fibroblast Activation Protein (FAP) Using the New Ligand [68Ga]Ga-OncoFAP-DOTAGA. European Journal of Nuclear Medicine and Molecular Imaging 2021. https://doi.org/10.1007/s00259-021-05653-0.

(7) Duan, H.; lagaru, A.; Aparici, C. M. Radiotheranostics - Precision Medicine in Nuclear Medicine and Molecular Imaging. Nanotheranostics 2022, 6 (1), 103-117. https://doi.org/10.7150/ntno.64141.

(8) Kratochwil, C.; Flechsig, P.; Lindner, T.; Abderrahim, L.; Altmann, A.; Mier, W.; Adeberg, S.; Rathke, H.; Röhrich, M.; Winter, H.; Plinkert, P. K.; Marme, F.; Lang, M.; Kauczor, H.-U.; Jäger, D.; Debus, J.; Haberkorn, U.; Giesel, F. L. 68Ga-FAPI PET/CT: Tracer Uptake in 28 Different Kinds of Cancer. Journal of Nuclear Medicine 2019, 60 (6), 801-805. https://doi.org/10.2967/jnumed.119.227967.

(9) Mona, C. E.; Benz, M. R.; Hikmat, F.; Grogan, T. R.; Lückerath, K.; Razmaria, A.; Riahi, R.; Slavik, R.; Girgis, M. D.; Carlucci, G.; Kelly, K. A.; French, S. W.; Czernin, J.; Dawson, D. W.; Calais, J. Correlation of 68Ga-FAPi-46 PET Biodistribution with FAP Expression by Immunohistochemistry in Patients with Solid Cancers: A Prospective Translational Exploratory Study. Journal of nuclear medicine: official publication, Society of Nuclear Medicine 2021. https://doi.org/10.2967/jnumed.121.262426.

(10) Jacopo, M.; Gabriele, B.; Jacqueline, M.; Abdullah, E.; Christian, P.; Aureliano, Z.; Sheila, D. P.; Lisa, N.; Andreas, G.; Eleonore, S.; Ilaria, B.; J, D. E.; Florent, S.; Dario, N.; Samuele, C. An Ultra-High-Affinity Small Organic Ligand of Fibroblast Activation 
Protein for Tumor-Targeting Applications. Proceedings of the National Academy of Sciences 2021, 118 (16), e2101852118. https://doi.org/10.1073/pnas.2101852118.

(11) Galbiati, A.; Zana, A.; Bocci, M.; Millul, J.; Elsayed, A.; Mock, J.; Neri, D.; Cazzamalli, S. A Novel Dimeric FAP-Targeting Small Molecule-Radio Conjugate with High and Prolonged Tumour Uptake. bioRxiv 2022, 2022.02.21.481260. https://doi.org/10.1101/2022.02.21.481260.

(12) Leal, M.; Wentland, J.; Han, X.; Zhang, Y.; Rago, B.; Duriga, N.; Spriggs, F.; Kadar, E.; Song, W.; McNally, J.; Shakey, Q.; Lorello, L.; Lucas, J.; Sapra, P. Preclinical Development of an Anti-5T4 Antibody-Drug Conjugate: Pharmacokinetics in Mice, Rats, and NHP and Tumor/Tissue Distribution in Mice. Bioconjugate Chemistry 2015, 26 (11), 2223-2232. https://doi.org/10.1021/acs.bioconjchem.5b00205.

(13) Sulochana, S. P.; Trivedi, R. K.; Srinivas, N. R.; Mullangi, R. A Concise Review of Bioanalytical Methods of Small Molecule Immuno-Oncology Drugs in Cancer Therapy. Biomedical Chromatography 2021, $35 \quad$ (1), e4996. https://doi.org/https://doi.org/10.1002/bmc.4996.

(14) Meermann, B.; Sperling, M. Hyphenated Techniques as Tools for Speciation Analysis of Metal-Based Pharmaceuticals: Developments and Applications. Analytical and Bioanalytical Chemistry 2012, 403 (6), 1501-1522. https://doi.org/10.1007/s00216012-5915-9.

(15) Miles, D. R.; Mesfin, M.; Mody, T. D.; Stiles, M.; Lee, J.; Fiene, J.; Denis, B.; Boswell, G. W. Validation and Use of Three Complementary Analytical Methods (LC-FLS, LCMS/MS and ICP-MS) to Evaluate the Pharmacokinetics, Biodistribution and Stability of Motexafin Gadolinium in Plasma and Tissues. Analytical and Bioanalytical Chemistry 2006, 385 (2), 345-356. https://doi.org/10.1007/s00216-006-0414-5.

(16) Miles, D.; Mody, T. D.; Hatcher, L. I.; Fiene, J.; Stiles, M.; Lin, P. P.; Lee, J. W. Quantitation of Motexafin Lutetium in Human Plasma by Liquid ChromatographyTandem Mass Spectrometry and Inductively Coupled Plasma-Atomic Emission Spectroscopy. AAPS PharmSci 2003, 5 (3), 1. https://doi.org/10.1208/ps050323.

(17) Coenen, H. H.; Elsinga, P. H.; Iwata, R.; Kilbourn, M. R.; Pillai, M. R. A.; Rajan, M. G. R.; Wagner, H. N.; Zaknun, J. J. Fluorine-18 Radiopharmaceuticals beyond [18F]FDG for Use in Oncology and Neurosciences. Nuclear Medicine and Biology 2010, 37 (7), 727-740. https://doi.org/https://doi.org/10.1016/j.nucmedbio.2010.04.185.

(18) Fersing, C.; Bouhlel, A.; Cantelli, C.; Garrigue, P.; Lisowski, V.; Guillet, B. A Comprehensive Review of Non-Covalent Radiofluorination Approaches Using Aluminum [18F]Fluoride: Will [18F]AIF Replace 68Ga for Metal Chelate Labeling? Molecules 2019, 24 (16). https://doi.org/10.3390/molecules24162866.

(19) Archibald, S. J.; Allott, L. The Aluminium-[18F]Fluoride Revolution: Simple Radiochemistry with a Big Impact for Radiolabelled Biomolecules. EJNMMI Radiopharmacy and Chemistry 2021, 6 (1), 30. https://doi.org/10.1186/s41181-02100141-0.

(20) Pacák, J.; Točík, Z.; Černý, M. Synthesis of 2-Deoxy-2-Fluoro-D-Glucose. Journal of the Chemical Society D: Chemical Communications 1969, No. 2, 77. https://doi.org/10.1039/C29690000077. 
(21) Liu, Y.; Hu, X.; Liu, H.; Bu, L.; Ma, X.; Cheng, K.; Li, J.; Tian, M.; Zhang, H.; Cheng, Z. A Comparative Study of Radiolabeled Bombesin Analogs for the PET Imaging of Prostate Cancer. Journal of Nuclear Medicine 2013, 54 (12), 2132. https://doi.org/10.2967/jnumed.113.121533.

(22) Blasi, F.; Oliveira, B. L.; Rietz, T. A.; Rotile, N. J.; Day, H.; Looby, R. J.; Ay, I.; Caravan, P. Effect of Chelate Type and Radioisotope on the Imaging Efficacy of 4 Fibrin-Specific PET Probes. Journal of Nuclear Medicine 2014, 55 (7), 1157. https://doi.org/10.2967/jnumed.113.136275.

(23) D'Souza, C. A.; McBride, W. J.; Sharkey, R. M.; Todaro, L. J.; Goldenberg, D. M. HighYielding Aqueous 18F-Labeling of Peptides via Al18F Chelation. Bioconjugate Chemistry 2011, 22 (9), 1793-1803. https://doi.org/10.1021/bc200175c. 


\section{Figures}

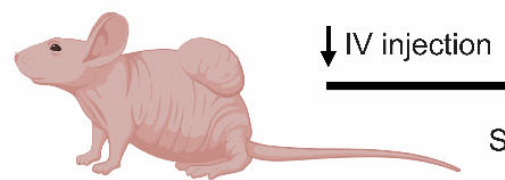

Tumor bearing mice $\mathrm{n}=3$

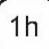

Sacrifice

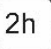

Sacrifice

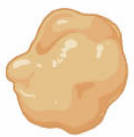

(tumor, heart, lung, liver, spleen, kidney, plasma)

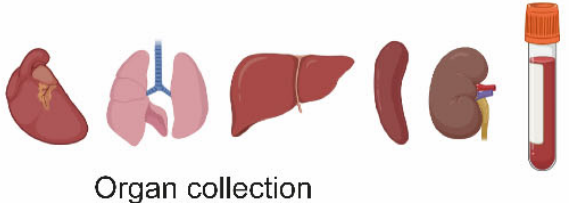

Organ collection
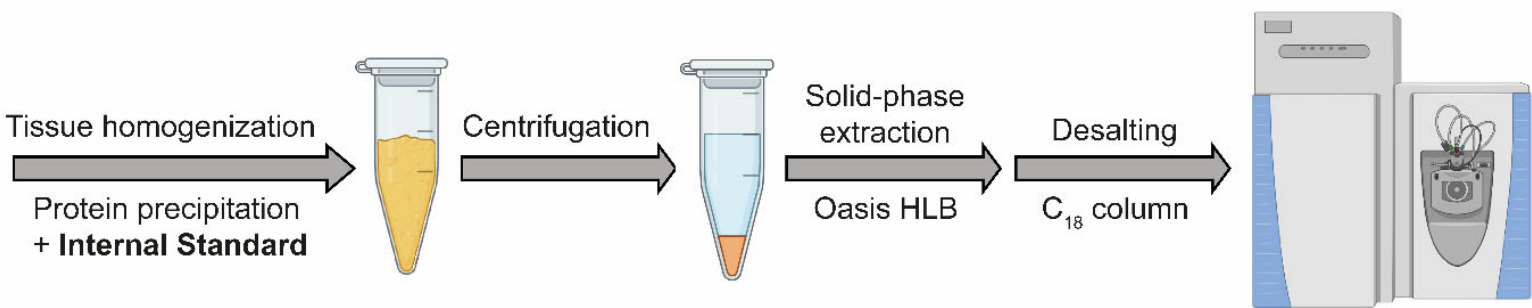

nanoLC/HRMS

Figure 1: Schematic workflow showing the experimental design and the sample preparation procedure. Mice $(n=3)$ were injected intravenously with different FAP-targeting compounds $(250 \mathrm{nmol} / \mathrm{Kg}$ or 500 $\mathrm{nmol} / \mathrm{Kg}$ ). Mice were sacrificed 1 or 2 hours post-injections, tissues were harvested and biospecimens processed and analyzed by nanoLC-HRMS. 


\section{FAP-targeting moieties}

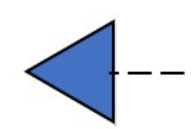<smiles>CC(=O)CCC(=O)Nc1cccc2c(C(=O)NCC(=O)N3CC(F)(F)CC3C#N)ccnc12</smiles>

OncoFAP
${ }^{13} \mathrm{C}_{4}$-OncoFAP

$\overbrace{\mathrm{CN}}^{\mathrm{F}}$

H<smiles>CCCCNC(=O)CCC(=O)Nc1cccc2c(C(=O)NCC)ccnc12</smiles><smiles>CC[C@@H](NC(=O)CCC(=O)Nc1cccc2c(C(=O)NCC(=O)N3CC(F)(F)C[C@H]3C#N)ccnc12)C(C)=O</smiles>

BiOnCOFAP

${ }^{13} \mathrm{C}_{6}{ }^{15} \mathrm{~N}_{2}$-BiOncoFAP

\section{Metal-chelator systems}<smiles>Cc1ccc(I)cc1</smiles>

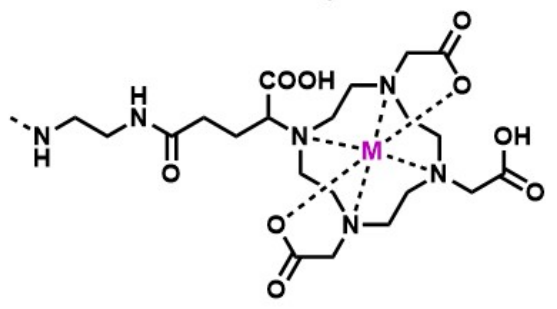

M-DOTAGA

$M=\left[{ }^{\text {nat }} L u\right] L u,\left[{ }^{\text {nat }} G a\right] G a$

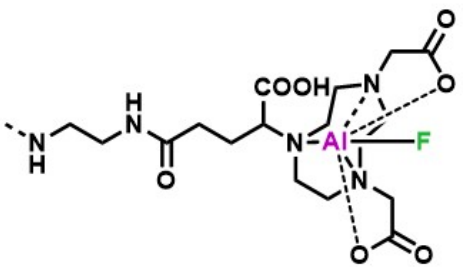

[ nat F]AIF-NODAGA

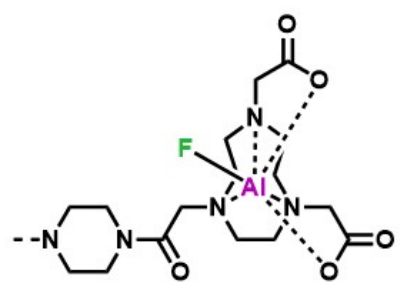

[ ${ }^{\text {nat }}$ F]AIF-NOTA

Figure 2: Chemical structures of targeting moieties (i.e., OncoFAP, and BiOncoFAP), and structures of metal chelators (i.e., DOTAGA, NODAGA, and NOTA) used in this study. Colored asterisks indicate the position of either ${ }^{13} \mathrm{C}$ or ${ }^{15} \mathrm{~N}$ incorporated in the internal standard. 

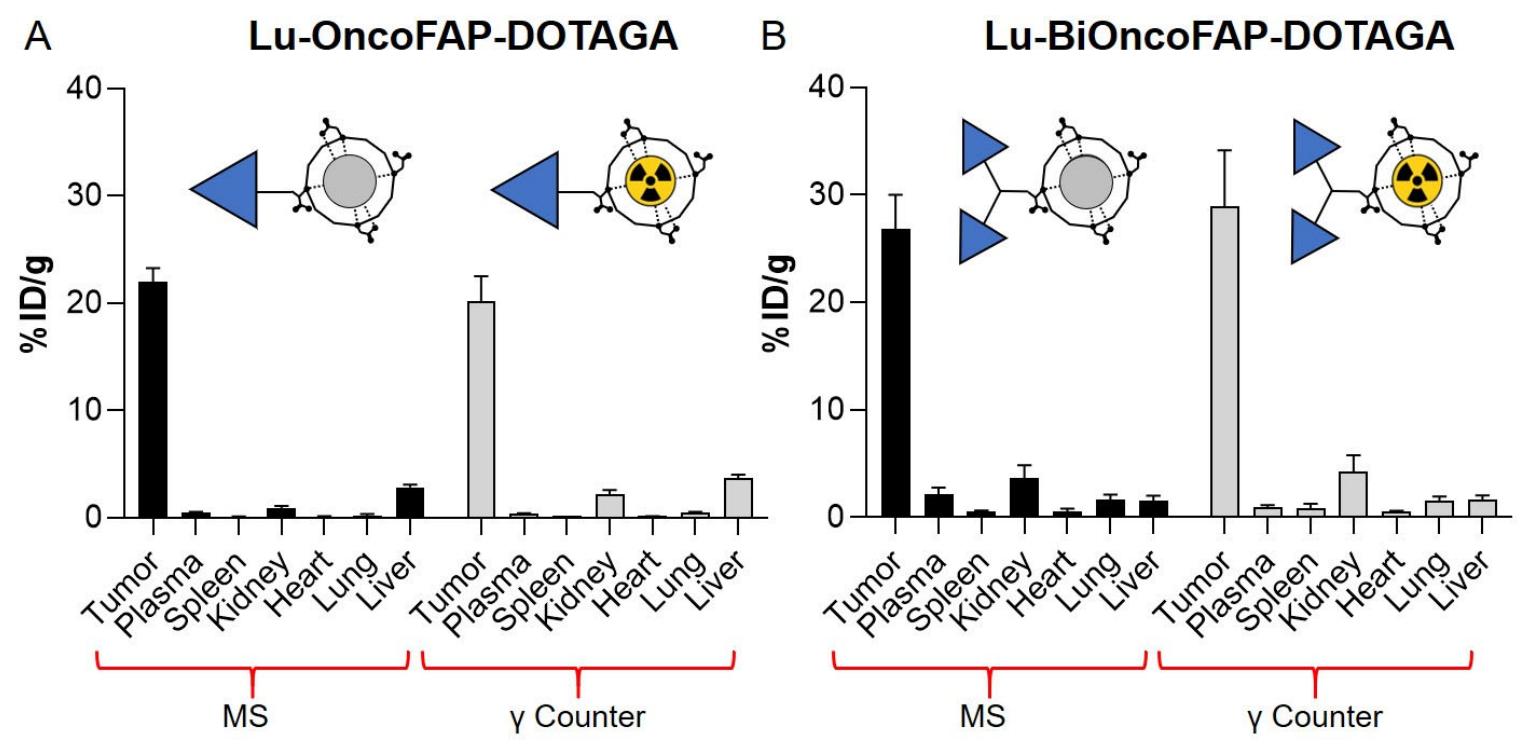

Figure 3: Comparison of MS and y Counter ${ }^{11}$ biodistribution data for $A$ ) Lu-OncoFAP-DOTAGA and $B$ ) Lu-BiOncoFAP-DOTAGA. Mice bearing HT-1080.hFAP tumors were sacrificed $1 \mathrm{~h}$ after intravenous administration $(250 \mathrm{nmol} / \mathrm{kg})$. No significant differences were observed between biodistribution results measured by MS and by radioactivity ( $p>0.05$, multiple $t$ test, table $\mathbf{S 4})$.

A

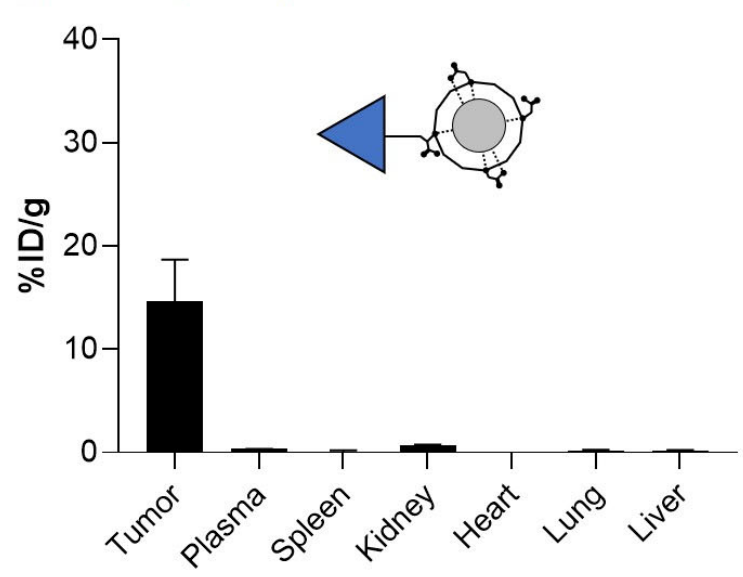

[ $\left.{ }^{\text {nat }} \mathrm{Ga}\right] \mathrm{Ga}-B i O n c o F A P-D O T A G A$

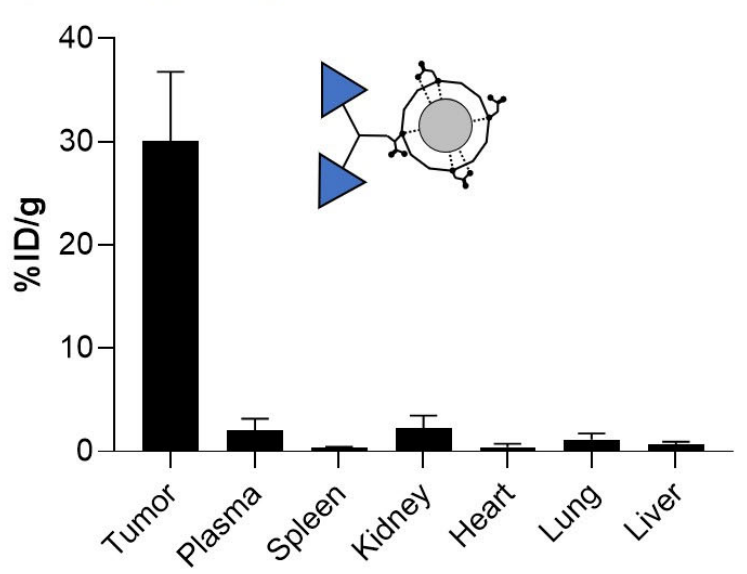

Figure 4:MS biodistribution results of $A$ ) [ $\left.{ }^{\text {nat }} \mathrm{Ga}\right] \mathrm{Ga}-$ OncoFAP-DOTAGA and B) [ $\left.{ }^{\text {nat }} \mathrm{Ga}\right] \mathrm{Ga}-\mathrm{BiOncoFAP-}$ DOTAGA. Mice bearing HT-1080.hFAP tumors were sacrificed $1 \mathrm{~h}$ after intravenous administration (250 $\mathrm{nmol} / \mathrm{kg})$. 
A

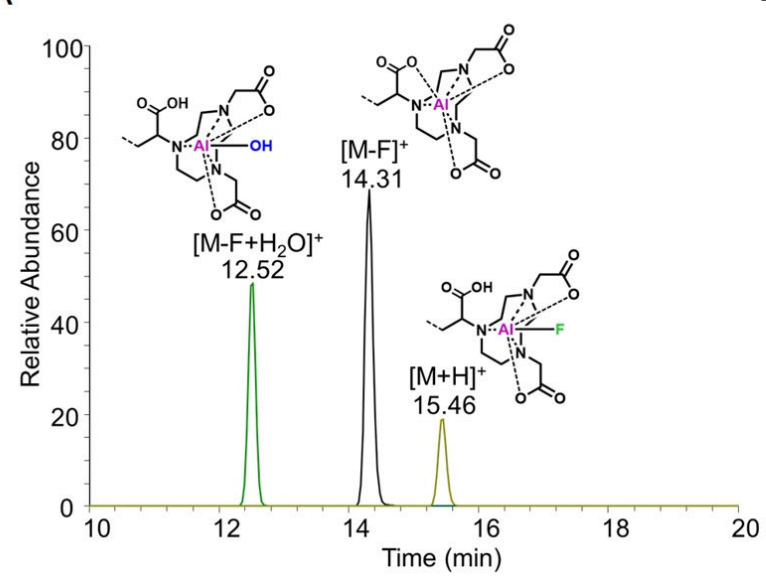

C

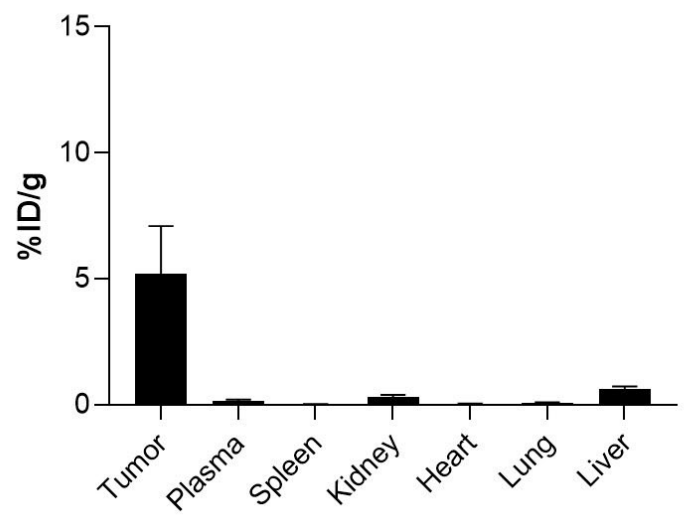

B

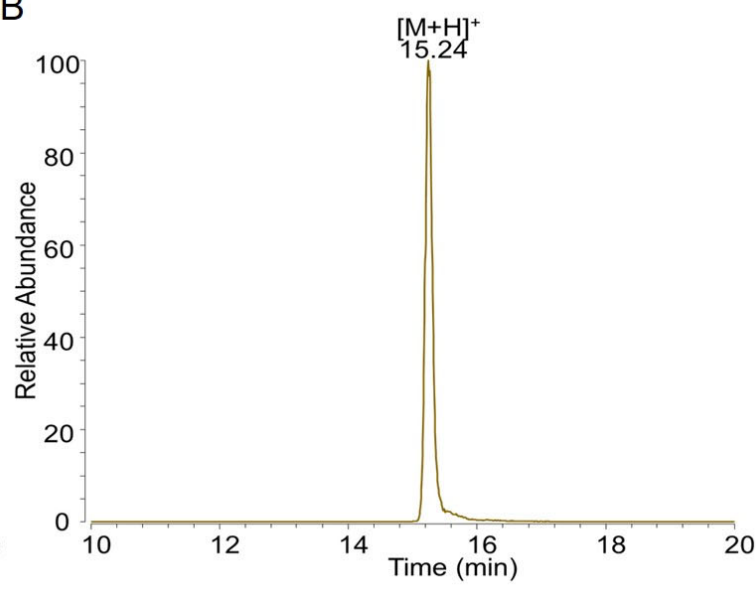

D [ [ ${ }^{\text {nat } F] A I F-O n c o F A P-N O T A ~}$

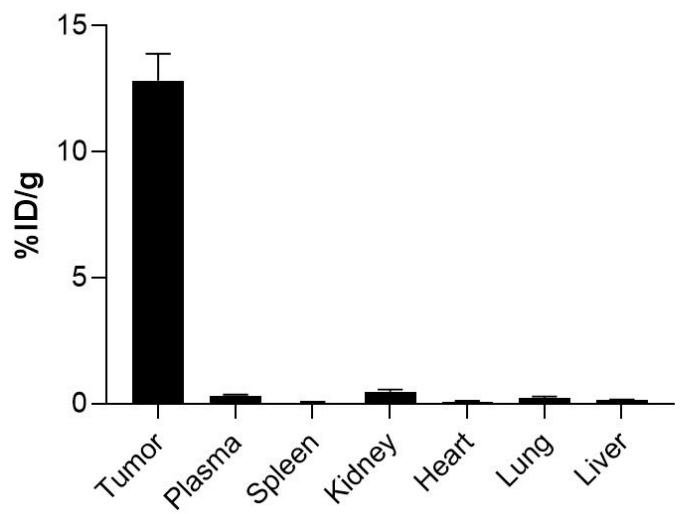

Figure 5: Chromatograms of A) OncoFAP-NODAGA and B) OncoFAP-NOTA, after labeling with Al ${ }^{\text {nat }} \mathrm{F}$; MS biodistribution results of $C$ ) OncoFAP-NODAGA and D) [natF]AIF-OncoFAP-NOTA. Chromatogram (A) evidences the presence of three different molecular species eluting at different retention times: AlOH-OncoFAP-NODAGA ( $\mathrm{Rt}=13.56 \mathrm{~min}$ ), Al-OncoFAP-NODAGA (Rt = $15.34 \mathrm{~min}$ ) and [ ${ }^{\text {natF]AIF- }}$ OncoFAP DOTAGA ( $\mathrm{Rt}=16.42 \mathrm{~min}$ ); The chromatogram of OncoFAP-NOTA $(\mathrm{B})$ shows the presence

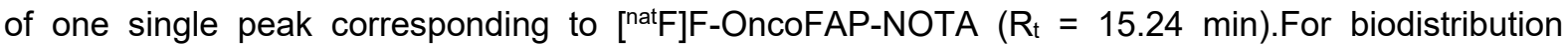
experiments (C-D) mice bearing SK-RC-52.hFAP tumors were sacrificed $2 \mathrm{~h}$ after intravenous administration (500 nmol/kg). For OncoFAP-NODAGA (C), the \%ID/g was calculated as sum of the three different molecular species (AIOH-OncoFAP-NODAGA, AI-OncoFAP-NODAGA and [natF]AIFOncoFAP DOTAGA) observed and measured by MS. For OncoFAP-NOTA (D) the \%ID/g refers to the only molecular species ([natF]F-OncoFAP-NOTA) measured by MS. 